\title{
INDUÇÃO DA OSTEOPOROSE SECUNDÁRIA POR VIA DE FÁRMACOS: UMA REVISÃO DE LITERATURA.
}

\section{Caroline Valduga Dozza1; Giovanna Teixeira Girardello²; Luísa Barbiero Dutra3; Maria Elizabeth Bonorino Bortolanza4; Rui Alberto Castilhos Ferreira Junior ${ }^{5}$; Rafaela Martinez Copês Leal ${ }^{6}$}

\section{RESUMO}

A osteoporose é definida como uma doença de redução da densidade óssea. De acordo com o Ministério da Saúde, aproximadamente 10 milhões de brasileiros são acometidos por osteoporose. Essa é uma doença de caráter sistêmico, associada ao metabolismo ósseo anormal e redução da densidade mineral óssea (DMO). Atualmente, no Brasil, vem ocorrendo uma maior tendência ao envelhecimento populacional, e, associado a isso, tem-se um aumento da polifarmácia, o que gera preocupações acerca dos riscos do desenvolvimento de osteoporose induzida por drogas (OID). A presente revisão de literatura apresenta a prevalência de associações de usos medicamentosos com o desenvolvimento de OID. Estes medicamentos incluem: antidepressivos, antiepiléticos, antipsicóticos, glicocorticoides, heparina, inibidores aromatase, inibidores da bomba de prótons e terapia de reposição tireoidiana.

Palavras-chave: Perda Óssea; Medicamentosa; Efeitos Adversos.

Eixo Temático: Atenção Integral e Promoção à Saúde (AIPS).

\section{INTRODUÇÃO}

Os ossos são um composto de materiais, os quais consistem na forma inorgânica (60\%), orgânica (32-30\%) e água (8-10\%) (ARCUS, Robert; et al). A fase predominante, inorgânica, é composta principalmente por cristais de hidroxiapatita $\left(\mathrm{Ca}_{10}\left[\mathrm{PO}_{4}\right]_{6}[\mathrm{OH}]_{2}\right)$, já a fase orgânica, por sua vez, é formada predominantemente por colágeno do tipo 1 e proteínas não colagenosas.

\footnotetext{
${ }^{1}$ Autor/Apresentador Acadêmica do Curso de Medicina - Universidade Franciscana. caroline.dozza@ufn.edu.br

2 Coautora Acadêmica do Curso de Medicina - Universidade Franciscana. giovanna.teixeira@ufn.edu.br

${ }^{3}$ Coautora Acadêmica do Curso de Medicina - Universidade Franciscana. luisa.dutra@ufn.edu.br

${ }^{4}$ Coautora Acadêmica do Curso de Medicina - Universidade Franciscana. maria.bortolanza@ufn.edu.br
} 
${ }^{5}$ Coautor Acadêmico do Curso de Medicina - Universidade Franciscana. rui.ferreira@ufn.edu.br 6 Orientadora Professora do Curso de Medicina - Universidade Franciscana.

rafaela.copes@ufn.edu.br

A homeostasia do tecido ósseo é regulada pelos osteoclastos, osteoblastos e osteócitos. Estes tipos celulares estão separados em dois grupos de linhagens celulares: a mesenquimal e a hematopoiética, as quais garantem a manutenção da homeostase óssea e as intimas interações entre o sistema imunológico e o sistema esquelético (ROSEN, Clifford J.).

Os componentes celulares da matriz são os responsáveis por manter um equilíbrio entre reabsorção, remodelação e formação, uma vez que este equilíbrio é rompido, ocorre uma redução na densidade e, consequentemente, um maior risco à fraturas. Isso se dá uma vez que a microarquitetura óssea possui uma relação direta com sua força.

O envelhecimento da população gera maiores riscos de polifarmácóia e, consequentemente, um maior risco de OID (O'CONNELL, Mary Beth et al.), sendo o uso de glicocorticoides o mais prevalente na indução da osteoporose (SALLÉS, Meritxell; GELMAN, Saúl Mario). O mecanismo e ação dos medicamentos, para o desenvolvimento de OID pode ocorrer de maneira direta, afetando a microarquitetura do osso, ou de maneira indireta, alterando o metabolismo e absorção de vitamina D e cálcio, que pode ser demasiadamente reduzido em alguns estados hormonais como o estresse oxidativo, hipogonadismo ou hipertireoidismo (AGGARWAL, Anshita et al.).

A osteoporose é classificada em duas categorias: primária (tipo 1), a é desencadeada principalmente pela queda do estrogênio no período pós-menopausa; e secundária (tipo 2), associada a outras doenças preexistentes, e o uso de determinados medicamentos na qual se insere a OID. Nesse contexto, destaca-se, ainda, que a osteoporose vem sendo encontrada em indivíduos sem predisposições genéticas ou influências ambientais (TANNIRANDORN, P.; EPSTEIN, S.).

\section{METODOLOGIA}

Para o desenvolvimento deste trabalho, realizou-se uma busca online por artigos presentes em uma das principais bases de dados (Portal de Periódicos da CAPES), incluindo literatura de qualquer ano de publicação. Neste processo de pesquisa, foram utilizados os descritores "osteoporosis"; "drug-induced".

Foram selecionados, neste processo, vinte e um (21) artigos, os quais apresentam os descritores mencionados. Destas obras, 12 são de, no máximo, 5 anos. Além disso, foram utilizados dois (02) livros didáticos como auxílio para a execução do texto. Assim, foi possível analisar fontes atuais acerca da temática da indução de osteoporose por uso de medicamentos, a fim de descrever, nesta revisão de literatura, o tema da maneira mais concisa possível. 


\section{RESULTADOS E DISCUSSÕES}

\subsection{Antidepressivos}

O aumento do risco de fraturas, devido ao uso de antidepressivos é elevado, principalmente em população idosa. Receptores e transportadores funcionais de serotonina (5-hidroxitriptamina [5-HT]) (5- HTT) foram identificados em osteoblastos, osteoclastos e osteócitos; no entanto, seus papéis na remodelação óssea não foram determinados. (O'CONNELL, Mary Beth).

Pesquisas recentes mostram uma associação entre o uso diário de inibidores seletivos da recaptação da serotonina e uma diminuição na DMO do quadril em mulheres, homens e idosos. Ademais de uma diminuição na DMO lombar nos homens. (SALLÉS, Meritxell; GELMAN, Saúl Mario).

\subsection{Antiepiléticos}

Os antiepiléticos podem causar perda óssea, mas seus mecanismos não estão completamente esclarecidos. Atualmente, existem várias teorias acerca de seu mecanismo de indução à OID. A maioria dos antiepiléticos provavelmente possuem relação com um metabolismo anormal da vitamina D (DREZNER, Marc K.; MAZZIOTTI, Gherardo; CANALIS, Ernesto;), além de efeitos inibitórios na diferenciação dos osteoblastos. O Valproato não apresenta efeitos na metabolização da vitamina D (DREZNER, Marc K.;), no entanto, apresenta efeitos antiandrogênicos (MAZZIOTTI, Gherardo; CANALIS, Ernesto;). A Fenitoína, por sua vez, eleva remodelação óssea, inibe a absorção de cálcio e a proliferação de osteoblastos (O'CONNELL, Mary Beth) e essa, como monoterapia, demonstrou reduzir a DMO em $1,2 \%$ por ano de tratamento. (SWEENEY, Brooke L.).

Estudos recentes com pacientes jovens em acompanhamento ambulatorial mostram uma diminuição da DMO na coluna lombar e no fêmur, mas não se correlaciona com os baixos níveis de vitamina $\mathrm{D}$. Esses resultados sugerem um efeito direto dos antiepilépticos na remodelação do tecido ósseo, no entanto, não conseguem identificar se algum medicamento é mais propício a desencadear OID que os demais. (SALLÉS, Meritxell; GELMAN, Saúl Mario).

Portanto, estes dados reforçam a importância da suplementação de cálcio e de vitamina $D$ para se evitar osteoporose, principalmente no que tange à OID induzida por antiepilépticos. É válido ressaltar, ainda, que há o aumento do risco de quedas e consequentes fraturas, associado a distúrbios do equilíbrio e efeito sedativo dos antiepiléticos. (BRIOT, Karine). 


\subsection{Antipsicóticos}

A ingestão de lítio a longo prazo está associada com um risco aumentado de hiperparatireoidismo, porém, não foram encontradas diferenças significativas quanto a redução de DMO (BRIOT, Karine.), no entanto, ainda há divergências com relação a esta afirmativa (O'CONNELL, Mary Beth et al.).

O mecanismo pelo qual os antipsicóticos afetam a fisiologia óssea está relacionado à estimulação da secreção de prolactina. Os antipsicóticos influenciam a secreção de prolactina por meio de seu antagonismo da dopamina endógena (DA) nos receptores DA-2 no cérebro, com maior afinidade para antipsicóticos convencionais, os quais estimulam a secreção de prolactina em maior grau em comparação aos antipsicóticos atípicos. A prolactina então reduz as concentrações de estrogênio e testosterona, resultando em hipogonadismo, que por sua vez reduz a DMO. (O'CONNELL, Mary Beth et al.).

\subsection{Glicocorticoides}

Glicocorticoides são os principais medicamentos indutores de OID, e a segunda principal causa de osteoporose, após a deficiência de estrogênio pós-menopausa (O'CONNELL, Mary Beth et al. ). É válido ressaltar que, algumas vezes, a doença a qual é tratada com glicocorticoides pode também ser um fator desencadeador de osteoporose primária (SALLÉS, Meritxell; GELMAN, Saúl Mario).

Os mecanismos de indução de OID por glicocorticoides são complexos, alterando a estrutura do osso, tanto diretamente quanto indiretamente, isso ocorre de maneira rápida, sendo a redução da DMO significativa no primeiro ano de tratamento. De início, ocorre um aumento da expressão do receptor-ativador nuclear kappa $B$ ligante (RANKL) e m-CSF. Concomitante a isto, ocorrerá uma redução na expressão do receptor de decodificação do RANKL, a osteoprotegerina (OPG), cessando a reabsorção óssea. Com um tempo longo de utilização dos medicamentos, ocorrerá um aumento da apoptose de osteoclastos e osteócitos (O'CONNELL, Mary Beth et al.; YANBEIY, Zeina A.; HANSEN, Karen E ).

Concatenado a isso, ocorre uma redução na maturação dos osteoblastos, o que ocasiona uma redução na formação óssea e, consequentemente, da DMO. A terapia com glicocorticoides também pode contribuir para a osteoporose, por causar hipogonadismo hipogonadotrófico, pela redução da absorção intestinal do cálcio e pela hipercalciúria (MIRZA, Faryal; CANALIS, Ernesto), ou seja, a indução à OID por glicocorticoides também pode ocorrer via hipogonadismo e hiperparatireoidismo secundários.

Estudos com a utilização de denosumabe na OID induzida por dexametasona demonstraram que aquela droga aumentou de maneira significativa a DMO na coluna 
lombar $(2.32 \%, 95 \% \mathrm{Cl} 1.73 \%, 2.91 \%, P<0.0001)$ e na região do quadril $(1.52 \%, 95 \%$ Cl 1.1\%,1.94\%, $P<0.0001$ ) quando comparado a biofosfonatos (YANBEIY, Zeina A.; HANSEN, Karen E).

Além disso, em meta-análise de 9 estudos randomizados foi constatado que pacientes com OID induzida por glicocorticoides, ao utilizarem alendronato apresentam um aumento de DMO da coluna vertebral e colo do fêmur $(P<0,00001)$ em comparação com a suplementação de cálcio, porém não foi demonstrada superioridade em relação a redução da fratura vertebral e não vertebral (WANG, YaKang et al.).

\subsection{Heparina}

O mecanismo da osteoporose induzida por heparina é multifatorial. O crescimento dos osteoblastos é prejudicado por meio da ligação competitiva das proteínas de ligação à superfície dos osteoblastos para evitar a ligação de fatores de crescimento importantes, como o IGF, que ajuda a estimular a replicação dos osteoblastos. Os efeitos da heparina na osteoporose são principalmente relacionados à dose. A dose crítica de heparina para ter um efeito adverso no osso parece ser mais de 15.000 unidades por dia por mais de 3 meses. (O'CONNELL, Mary Beth).

A heparina diminui a formação do osso e aumenta a reabsorção, esta última por inibir a expressão da osteoprotegerina, um receptor chamariz para o receptor ativador do fator nuclear - ligante kappa B. (MAZZIOTTI, Gherardo et al.).

A pesquisa mostrou que a vitamina $\mathrm{K}$ desempenha um papel no metabolismo ósseo e é potencialmente protetora contra a osteoporose. A vitamina $\mathrm{K}$ é necessária para a gama carboxilação da osteocalcina, que é uma proteína óssea. Os anticoagulantes à base de cumarina que interferem neste processo. (SWEENEY, Brooke L.).

Estudos recentes observaram que o risco de desenvolver osteoporose é menor quando a heparina de baixo peso molecular é usada em comparação com a heparina não fracionada. Estudos em animais sugerem que a heparina não fracionada causa perda de DMO, causando diminuição da formação e aumento da reabsorção do osso, enquanto a heparina de baixo peso molecular apenas produz uma diminuição na formação óssea (SALLÉS, Meritxell; GELMAN, Saúl Mario), ao contrário do anticoagulante Fondaparinux, criado como uma alternativa à heparina (MAZZIOTTI, Gherardo; CANALIS, Ernesto;).

\subsection{Inibidores da aromatase}

Aromatase é uma enzima da via biossintética convertendo andrógenos adrenais. (TANNIRANDORN, P.; EPSTEIN, S.). O mecanismo de ação destes 
medicamentos se baseia na inibição da conversão de androgênios em estrogênios resultando em baixos níveis de estrogênios basais (PANDAY, Keshav; GONA, Amitha; HUMPHREY, Mary Beth.), sendo mais utilizado por mulheres pós-menopausa e em tratamento do câncer de mama (MAZZIOTTI, Gherardo et al.; O'CONNELL, Mary Beth et al.) As quais já possuem em si um fator de risco isolado para o desenvolvimento de OID.

Além disso, vale rememorar que, medicamentos utilizados no período prémenopausa podem causar uma perda de DMO irreversível (BINKLEY, N. et al.). O ácido zoledrônico e o risedronato mostraram prevenir e reverter a perda óssea, mas os dados sobre a eficácia da redução da fratura são escassos. (MAZZIOTTI, Gherardo).

\subsection{Inibidores da bomba de prótons}

Estudos observacionais sugerem que os inibidores de bomba de prótons possuem uma associação com o aumento de risco de fraturas osteoporóticas (Yang et al.), induzindo uma redução de DMO na coluna lombar e no quadril, e aumentam o risco de fraturas por fragilidade vertebral e não vertebral, dependendo da dose do medicamento e da duração da terapia. (MAZZIOTTI, Gherardo; CANALIS, Ernesto; GIUSTINA, Andrea.). Por outro lado, demais estudos não demonstram diferenças significativas (PANDAY, Keshav; GONA, Amitha; HUMPHREY, Mary Beth.). Os mecanismos de indução da osteoporose por esta classe farmacológica seguem incertos.

Em ratos, os inibidores da bomba de prótons podem restringir a ação a bomba de prótons $\mathrm{H}+$ ATPase no osteoclasto, reduzindo, assim a reabsorção óssea; entretanto, esse efeito é 100 vezes menor do que a atividade na parede intestinal. Provavelmente, as doses de IBP humano são muito baixas para quaisquer efeitos ósseos positivos, conforme sugere BINKLEY, N. et al.

Ensaios recentes sugerem um risco aumentado de fratura osteoporótica em pacientes em tratamento crônico com IBP 54-56. Esses estudos são observacionais e randomizados são necessários para relacionar causalmente a diminuição na absorção de cálcio com a diminuição na DMO e o aumento nas fraturas (BRIOT, Karine.).

\subsection{Terapia de reposição tireoidiana}

A tiroxina é prescrita para o tratamento de hipotireoidismo, bócio e carcinoma da tireoide após ablação de tireoide. No entanto, apenas uma sobredose pode levar a perda óssea clinicamente relevante com aumento da reabsorção do osso (BARTL, Reiner; BARTL, Ch; GRADINGER, Robert.) e, consequentemente, a uma redução da DMO de 10 a 12\% (O'CONNELL, Mary Beth et al.). No entanto, de acordo com BRIOT, 
Karine, o uso prolongado de medicamentos de reposição tireoidiana somente irá causar redução de DMO caso os níveis de TSH estiverem anormais.

O hipertireoidismo e a suplementação excessiva de hormônio tireoidiano foram associados ao aumento da perda óssea e fraturas devido ao aumento da reabsorção do tecido ósseo. A alta renovação do tecido ósseo, resultado da tireotoxicose relacionada ao aumento da ativação de osteoclastos e RANKL juntamente com aumento de citocinas, como TNF uma e IL-6. (O'CONNELL, Mary Beth et al.). Todavia, enquanto alguns estudos concluem que a terapia com L-tiroxina em doses supressivas pode produzir uma diminuição na DMO, especialmente em mulheres na pós-menopausa (diminuição não uniforme e mais rápida em 6 meses após o início do tratamento), outros não mostram diferenças significativas com o grupo de controle (SALLÉS, Meritxell; GELMAN, Saúl Mario.).

\section{CONCLUSÃO}

A osteoporose é uma doença a qual afeta uma grande parcela da população, ocorrendo de forma direta (pela queda de estrogênios no período pós-menopausa), ou de forma indireta, onde se encontra a OID. Tendo em vista os inúmeros tratamentos medicamentosos utilizados na prática clínica, é necessário iniciar planos preventivos contra OID, principalmente de idosos e portadores, os quais correm maior risco de aderirem ao consumo de múltiplos fármacos.

Ainda não há certeza de que a suplementação de cálcio e vitamina $D$ auxiliam totalmente no tratamento de osteoporose e ainda não há estudos conclusivos quanto à recuperação total de DMO reduzida por drogas, a exemplo do denosumabe. Esta droga se mostrou mais promissora no tratamento de OID do que os biofosfanatos, atualmente utilizado. Além disso, a prevenção das quedas, e assim, de um maior risco de fraturas pelos portadores de osteoporose é um fator a ser considerado como relevante em orientações da prática clínica.

Sabe-se que os mecanismos de indução da OID variam de acordo com os medicamentos utilizados, porém, esta linha de pesquisa ainda carece de informações. De acordo com os meios de indução da OID, esses podem ser classificados como diretos, alterando diretamente a matriz do osso; indiretos, afetando de maneira indireta a densidade mineral óssea, por via de mecanismos como o hipogonadismo, hiperparatireoidismo; ou mistos.

\section{AGRADECIMENTOS}

Agradecimentos ao Curso de Medicina da Universidade Franciscana. 


\section{REFERÊNCIAS}

AGGARWAL, Anshita et al. Drug-induced bone disorders: A systematic review. Indian Journal of Rheumatology, v. 14, n. 5, p. 44, 2019.

BINKLEY, N. et al. Is drug-induced bone loss acceptable in premenopausal women? A practical fracture risk modeling exercise. Osteoporosis International, v. 28, n. 12, p. 3501-3513, 2017.

BARTL, Reiner; BARTL, Ch; GRADINGER, Robert. Medikamenteninduzierte Osteopathien. Zeitschrift für Rheumatologie, v. 69, n. 2, 2010.

BRIOT, Karine. Ostéopathies médicamenteuses (hors cortisone). La Presse Médicale, v. 35, n. 10, p. 1579-1583, 2006.

DREZNER, Marc K. Treatment of anticonvulsant drug-induced bone disease. Epilepsy \& Behavior, v. 5, p. 41-47, 2004.

FERREIRA, Davilson Bragine et al. Perfil biométrico, histomorfométrico e bioquímico no tratamento com atorvastatina cálcica de ratas com osteoporose induzida com dexametasona. Revista brasileira de ortopedia, v. 53, p. 607-613, 2018.

LIN, Hao et al. Sulforaphane reverses glucocorticoid-induced apoptosis in osteoblastic cells through regulation of the Nrf2 pathway. Drug design, development and therapy, v. 8, p. 973, 2014.

MARCUS, Robert; FELDMAN, David; NELSON, Dorothy A.; ROSEN, Clifford J. Osteoporosis. Elsevier, v.3 p. 14-36, 2010.

MAZZIOTTI, Gherardo; CANALIS, Ernesto; GIUSTINA, Andrea. Drug-induced osteoporosis: mechanisms and clinical implications. The American journal of medicine, v. 123, n. 10, p. 877-884, 2010.

MIRZA, Faryal; CANALIS, Ernesto. Secondary osteoporosis: pathophysiology and management. European journal of endocrinology/European Federation of Endocrine Societies, v. 173, n. 3, p. R131, 2015.

O'CONNELL, Mary Beth et al. Drug-induced osteoporosis in the older adult. Aging health, v. 6, n. 4 , p. 501-518, 2010.

PANDAY, Keshav; GONA, Amitha; HUMPHREY, Mary Beth. Medication-induced osteoporosis: screening and treatment strategies. Therapeutic advances in musculoskeletal disease, v. 6, n. 5, p. 185-202, 2014. 
QIN, Zinan et al. Combination therapy of wuweizi (Schisandrae Chinensis Fructus) and dexamethasone alleviated dexamethasone-induced glucocorticoid osteoporosis in rats with idiopathic pulmonary fibrosis. BioMed research international, v. 2020, 2020.

ROSEN, Clifford J. Primer on the metabolic bone diseases and disorders of mineral metabolism. John Wiley \& Sons, 2009.

SHAPIRO, Charles L. Osteoporosis: a long-term and late-effect of breast cancer treatments. Cancers, v. 12, n. 11, p. 3094, 2020.

SALLÉS, Meritxell; GELMAN, Saúl Mario. Osteoporosis secundaria a fármacos. Seminarios de la Fundación Española de Reumatología, v. 10, n. 4, p. 112-117, 2009.

SWEENEY, Brooke L. Drug-induced bone disorders. Clinical Reviews in Bone and Mineral Metabolism, v. 3, n. 1, p. 75-92, 2005.

SHI, Lianjie et al. Technetium-99 Conjugated with Methylene Diphosphonate Ameliorates Glucocorticoid Induced Osteoporosis by Inhibiting Osteoclastogenesis. BioMed research international, v. 2018, 2018.

TANNIRANDORN, P.; EPSTEIN, S. Drug-induced bone loss. Osteoporosis international, v. 11, n. 8, p. 637-659, 2000.

WANG, Junjie; LI, Hongzhuo. Treatment of glucocorticoid-induced osteoporosis with bisphosphonates alone, vitamin $\mathrm{D}$ alone or a combination treatment in eastern Asians: a meta-analysis. Current pharmaceutical design, v. 25, n. 14, p. 1653, 2019.

WANG, Ya-Kang et al. Effects of alendronate for treatment of glucocorticoid-induced osteoporosis: a meta-analysis of randomized controlled trials. Medicine, v. 97, n. 42, 2018.

YANBEIY, Zeina A.; HANSEN, Karen E. Denosumab in the treatment of glucocorticoidinduced osteoporosis: a systematic review and meta-analysis. Drug design, development and therapy, v. 13, p. 2843, 2019. 